\title{
Association of homocysteine level with biopsy- proven non-alcoholic fatty liver disease: a meta-analysis
}

\author{
Yining Dai, ${ }^{1,+}$ Jinzhou Zhu, ${ }^{1,+}$ Di Meng, ${ }^{2}$ Chaohui Yu${ }^{1}$ and Youming Li ${ }^{1, *}$ \\ 'Department of Gastroenterology and '2Department of Thoracic Surgery, The First Affiliated Hospital, College of Medicine, Zhejiang University, \\ Hangzhou, 310003, Zhejiang, China
}

(Received 15 April, 2015; Accepted 1 June, 2015; Published online 29 August, 2015)

\begin{abstract}
Previous studies have reported inconsistent findings regarding the association between plasmatic higher of homocysteine level and non-alcoholic fatty liver disease. We aimed to investigate this association by conducting a meta-analysis. Literature was searched on PubMed from inception to January 2015. Eight studies evaluating plasma level of homocysteine in biopsy-proven non-alcoholic fatty liver disease subjects compared to healthy controls were included. Compared with the controls, non-alcoholic fatty liver disease patients witnessed a higher level of homocysteine [standard mean difference (SMD): $0.66 \mu \mathrm{mol} / \mathrm{L}, 95 \% \mathrm{Cl}: 0.41,0.92 \mu \mathrm{mol} / \mathrm{L}]$, and were associated with a significant increased risk for hyperhomocysteinemia [odds ratio (OR) $5.09,95 \% \mathrm{Cl}: 1.69,15.32$ ]. In addition, patients with non-alcoholic fatty liver presented $0.45 \mu \mathrm{mol} / \mathrm{L}$ higher levels of homocysteine compared to healthy controls ( $95 \% \mathrm{Cl}: 0.09,0.82 \mu \mathrm{mol} / \mathrm{L})$, whereas non-alcoholic steatohepatitis patients had $1.02 \mu \mathrm{mol} / \mathrm{L}$ higher levels of homocysteine (95\% Cl: $0.28,1.76 \mu \mathrm{mol} / \mathrm{L}$ ). There was neither difference of folate level nor vitamin B12 level between non-alcoholic fatty liver disease subjects and healthy controls. This study revealed that non-alcoholic fatty liver disease patients presented an increased serum concentration of homocysteine, and were associated with an increased risk of hyperhomocysteinemia. Further studies are needed to demonstrate a causal role of hyperhomocysteinemia in non-alcoholic fatty liver disease.
\end{abstract}

Key Words: non-alcoholic fatty liver disease, homocysteine, hyperhomocysteinemia, folate, vitamin B12

$\mathrm{N}$ on-alcoholic fatty liver disease (NAFLD) is one of the most common chronic liver diseases worldwide, affecting approximate $30 \%$ of the general adult population. ${ }^{(1)}$ NAFLD comprises of a wide spectrum of liver damage ranging from non-alcoholic fatty liver (NAFL) to non-alcoholic steatohepatitis (NASH), to fibrosis and cirrhosis that can progress to liver failure and hepatocellular carcinoma. ${ }^{(2)}$ Liver biopsy has long been taken as the gold standard for characterizing liver histology in patients with NAFLD. ${ }^{(1)}$ Nowadays, NAFLD is increasingly recognized as the hepatic manifestation of insulin resistance (IR) and metabolic syndrome, which is characterized by obesity, type 2 diabetes mellitus (T2DM), dyslipidemia and hypertension. ${ }^{(3)}$

Homocysteine (Hcy), formed as an intermediary in methionine metabolism, is a sulfur-containing amino acid. Hcy is biosynthesized from methionine via a multi-step process. According to the former studies, its metabolism consists of two pathways: remethylation to methionine, which requires folate and vitamin B12 coenzymes; and transsulfuration to cysteine, which requires pyridoxal-5'-phosphate, a vitamin B6 coenzyme. ${ }^{(4)}$ An abnormally elevated level of Hcy in the serum was defined as hyperhomo- cysteinemia that has been proved to be a significant risk factor for cardiovascular disease and cerebrovascular disease. ${ }^{(5-7)}$ Moreover, it is potentially linked to increasing risk of fracture, ${ }^{(8)}$ the developments of T2DM ${ }^{(9)}$ and (micro)albuminuria. ${ }^{(10)}$

The liver plays a central role in the metabolism of methionine and Hcy. It has been reported that hyperhomocysteinemia alters intracellular lipid metabolism and changes in Hcy metabolism, which may occur in liver damage. ${ }^{(1-13)}$ There were some studies previously suggested that higher Hcy levels existed in NAFLD patients, while some other research reported serum Hcy concentrations were not associated with NAFLD. Thus we conducted a meta-analysis to estimate whether Hcy level is elevated in NAFLD patients compared to healthy controls, as well as the different levels of folate and vitamin B12 between NAFLD patients and healthy controls. Meanwhile, we evaluated the risk of hyperhomocysteinemia among NAFLD population.

\section{Materials and Methods}

Data sources and searches. The searching, identification and protocol description were based on the preferred reporting items for Systemic Reviews and Meta-Analyses (PRISMA) guidelines. ${ }^{(14)}$ Literature was searched on PubMed from inception to January 2015 with the following terms: (NAFLD OR NASH OR non-alcoholic fatty liver disease OR non-alcoholic steatohepatitis OR fatty liver OR steatohepat*) AND (homocysteine OR homocystine OR hyperhomocysteinemia OR folic acid OR folate OR vitamin B12 OR vitamin B6 OR pyridoxine). No language restriction was imposed.

Selection criteria. Two authors (YN Dai and D Meng) independently assessed the potentially eligible records with the following inclusion criteria: 1) original observational studies enrolling adult (age $\geq 18$ years) population of any sex or ethnicity; 2) providing data dealing with plasma Hcy level and/or the level of Hcy-related vitamins (folate, vitamin B12 or vitamin B6) and NAFLD; 3) NAFLD had to be ascertained by histology through liver biopsy, which is the golden standard for diagnosis. Competing etiologies for steatosis, including alcohol consumption, viral hepatitis infection, drug-induced liver disease, autoimmune hepatitis, primary biliary cirrhosis, primary sclerosing cholangitis and hereditary disorders had to be excluded. Patients with liver cirrhosis were also excluded; 4) Healthy controls had no evidence of liver disease by imaging and biochemical evaluation.

These authors contributed equally to this work *To whom correspondence should be addressed. E-mail: li_youming1956@163.com 
Data extraction. Data were abstracted independently based on the meta-analysis of observational studies in epidemiology (MOOSE) guideline. Disagreements on study eligibility and data abstraction were resolved depending on the third reviewer's opinion (JZ Zhu). Data were extracted independently by two reviewers (YN Dai and D Meng). The following information was abstracted from each included paper: first author's name, year of publication, location, number of cases and controls, gender, age, study design, baseline information of the enrolled subjects, mean values and standard deviation (SD) of fasting plasma Hcy, folate, vitamin B12 level in case and control group, the histological degree of NAFLD (if provided), the number of subjects exhibiting hyperhomocysteinemia (if provided).

Quality assessment. Methodological quality of studies included in the meta-analyses was evaluated by the 22-item STROBE score ${ }^{(15)}$ by two reviewers (YN Dai and D Meng), with the following two extra items added to the checklist: blinding of pathologists assessing liver biopsy; liver biopsy performed and scored referring to standard criteria. ${ }^{(1)}$

Categories of NAFLD. The degree of NAFLD was categorized into NAFL and NASH, according to the criteria of NAFLD activity score (NAS). NAS of $\geq 5$ correlated with a definition of $\mathrm{NASH}$, and biopsies with scores of less than 4 were defined as NAFL. ${ }^{(16)}$

Statistical analysis. Meta-analyses were performed using Hcy levels, as well as folate levels and vitamin B12 levels as primary outcomes. The continuous outcome measures were presented as standard mean difference (SMD) with $95 \%$ confidence intervals (CI). Statistical heterogeneity was assessed by the Cochran Q test, and the I-squared statistic. Heterogeneity was considered significant by the Cochran $\mathrm{Q}$ test with $p<0.05$ or by I-squared greater than $50 \%$. $^{(17,18)} \mathrm{A}$ fixed-effects model or random effects model was adopted, depending on the absence or presence of heterogeneity. Subgroup analyses were conducted. We examined the overall combined estimates for the level of Hcy and its relative vitamins separately for study types (cross-sectional studies and case-control studies). In order to identity whether Hcy concentrations change with the degree of NAFLD, we also examined the overall Hcy levels separately in NAFL patients and NASH patients compared to controls.

For studies available for the prevalence of hyperhomocysteinemia, we estimated the unadjusted odds ratio (OR) and $95 \%$ CI by creating a $2 \times 2$ table of NAFLD and controls by Hcy status. On the account of limited data, we contacted the authors of each study via emails for original data regarding Hcy level in each subject in NAFLD and control groups. OR was pooled using inverse variance method with a fixed-effects model.

Additionally, sensitivity analyses were performed to evaluate the influence of each study on the pooled measures by omitting one in each turn and then calculating the summarized SMDs for the remainders. Funnel plots ${ }^{(19)}$ were used initially to assess publication bias and later publication bias was confirmed using Egger's ${ }^{(20)}$ and Begg's tests. ${ }^{(21)}$ We conducted the meta-analyses using Stata Statistical Software (ver. 12.0; StataCorp LP, College Station, TX).

\section{Results}

Study selection, characteristics and quality. The selection process for the studies included in this meta-analysis was shown in Fig. 1. The initial search yielded 552 items. After each publication was screened through titles and abstracts, 524 of these were excluded due to non-human studies, irrelevant topics, not reporting primary data (reviews) and irrelevant population (alcoholic fatty liver disease). Following the reviewing of the full texts, a total of 8 studies were identified for inclusion.

The principal characteristics of the trials included in the metaanalyses, as well as the quality score of each study, were exhibited

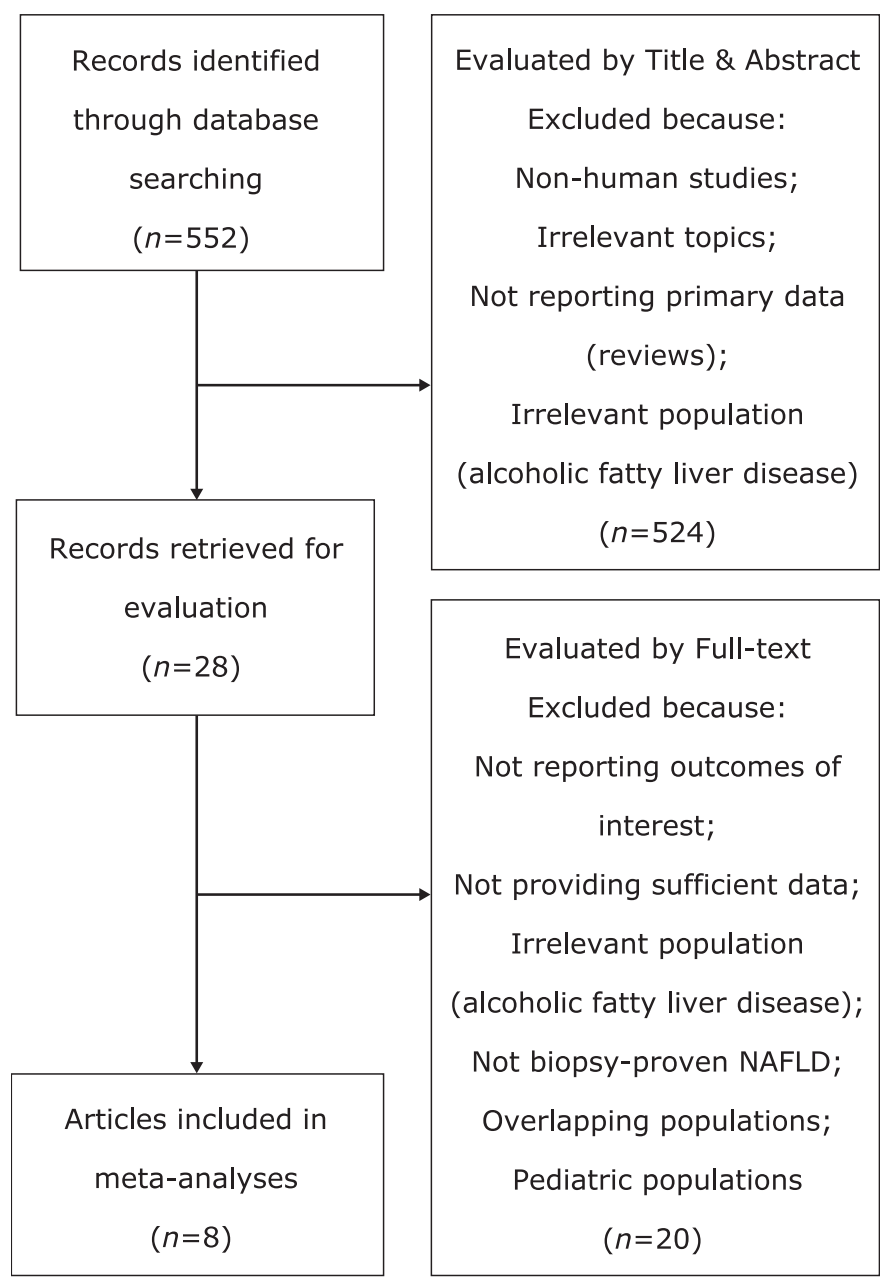

Fig. 1. Flow chart of the study selection process.

in Table 1. Six cross-sectional studies ${ }^{(22-27)}$ and two case-control study ${ }^{(28,29)}$ totaling 430 biopsy-proven NAFLD participants and 563 controls were identified. Overall, the methodological quality of the included studies was good: the median (range) STROBE score was 22.5 (20-23). Outcome measures of each study were listed in Table 2.

Hcy level. The meta-analysis for the levels of Hcy by NAFLD status included eight studies ${ }^{(22-29)}$ totaling 935 participants (397 NAFLD cases and 538 controls). Compared with the controls, in general, NAFLD patients witnessed a higher level of Hcy (SMD: $0.66 \mu \mathrm{mol} / \mathrm{L}, 95 \%$ CI: $0.41,0.92 \mu \mathrm{mol} / \mathrm{L}$ ). And there was a statistically significant heterogeneity among studies $\left(I^{2}=64.3 \%, p<0.01\right)$.

Based on types of studies, subgroup analysis revealed that NAFLD patients had $0.61 \mu \mathrm{mol} / \mathrm{L}$ higher levels of Hcy compared to the controls $(95 \% \mathrm{CI}: 0.25,0.97 \mu \mathrm{mol} / \mathrm{L})\left(I^{2}=72.8 \%, p<0.01\right)$ in six cross-sectional studies, ${ }^{(22-27)}$ while the SMD for Hcy level was $0.73 \mu \mathrm{mol} / \mathrm{L} \quad(95 \%$ CI: $0.47,0.98 \mu \mathrm{mol} / \mathrm{L}) \quad\left(I^{2}=1.8 \%\right.$, $p=0.313$ ) in two case-control studies (Fig. 2). ${ }^{(28,29)}$

As far as the degree of NAFLD was concerned, Fig. 3 indicated that patients with NAFL presented $0.45 \mu \mathrm{mol} / \mathrm{L}$ higher levels of Hcy when compared to healthy controls (95\% CI: 0.09 , $0.82 \mu \mathrm{mol} / \mathrm{L})\left(I^{2}=0.0 \%, p=0.618\right)$, whereas NASH patients had $1.02 \mu \mathrm{mol} / \mathrm{L}$ higher levels of Hcy $(95 \% \mathrm{CI}: 0.28,1.76 \mu \mathrm{mol} / \mathrm{L})$ $\left(I^{2}=91.0 \%, p<0.01\right)$ (Fig. 4).

Two studies ${ }^{(26,27)}$ enrolling 66 NAFLD cases and 47 controls provided us the original data regarding Hcy level in each subject. 
Table 1. Characteristics of studies included in the meta-analyses

\begin{tabular}{|c|c|c|c|c|c|c|c|c|}
\hline \multirow{2}{*}{ Study, year } & \multirow{2}{*}{ Country } & \multicolumn{2}{|c|}{$\begin{array}{l}\text { Number [Male/female, } \\
\text { mean age (years)] }\end{array}$} & \multirow{2}{*}{ Study design } & \multicolumn{2}{|c|}{ Categories of NAFLD } & \multirow{2}{*}{ Basline information } & \multirow{2}{*}{$\begin{array}{l}\text { Quality score } \\
\text { (falling items) }\end{array}$} \\
\hline & & Case & Control & & $\begin{array}{c}\text { NAFL } \\
\text { (number) }\end{array}$ & $\begin{array}{c}\text { NASH } \\
\text { (number) }\end{array}$ & & \\
\hline $\begin{array}{l}\text { de Carvalho SC, } \\
2013^{(22)}\end{array}$ & Brazil & $\begin{array}{c}35 \\
(9 / 26,49)\end{array}$ & $\begin{array}{c}51 \\
(16 / 35,39)\end{array}$ & $\begin{array}{l}\text { Cross-sectional } \\
\text { study }\end{array}$ & NA & NA & $\begin{array}{l}\text { BMI, AST, ALT, GGT, AP, insulin, HOMA- } \\
\text { IR, TG higher in case than control; HDL-c } \\
\text { lower in case than control; total-c, LDL-C, } \\
\text { FBG similar in case and control. }\end{array}$ & $(\mathrm{Q}, \mathrm{Y})$ \\
\hline $\begin{array}{l}\text { Franco Brochado MJ, } \\
2013^{(23)}\end{array}$ & Brazil & $\begin{array}{c}134 \\
(57 / 77,44)\end{array}$ & $\begin{array}{c}134 \\
(41 / 93,32)\end{array}$ & $\begin{array}{l}\text { Cross-sectional } \\
\text { study }\end{array}$ & NA & NA & $\begin{array}{l}\text { BMI, AST, ALT, GGT, AP, insulin, HOMA- } \\
\text { IR, FBG, total-c, LDL-C, TG, ferritin higher } \\
\text { in case than control; HDL-c, iron lower in } \\
\text { case than control. }\end{array}$ & $\begin{array}{l}22 \\
(J, Y)\end{array}$ \\
\hline Gulsen M, $2005^{(24)}$ & Turkey & $\begin{array}{c}71 \\
(61 / 10,38)\end{array}$ & $\begin{array}{c}30 \\
(26 / 4,39)\end{array}$ & $\begin{array}{l}\text { Cross-sectional } \\
\text { study }\end{array}$ & 23 & 36 & $\begin{array}{l}\text { AST, ALT, insulin, c-peptide, FBG, HOMA- } \\
\text { IR, total-C, TG, VLDL-c higher in case than } \\
\text { control; HDL-c lower in case than control; } \\
\text { LDL-C, BMI, Cr similar in case and control. }\end{array}$ & $\begin{array}{l}23 \\
(Y)\end{array}$ \\
\hline Hirsch S, $2005^{(25)}$ & Chile & $\begin{array}{c}17 \\
(0 / 17,37)\end{array}$ & $\begin{array}{c}26 \\
(0 / 26,42)\end{array}$ & $\begin{array}{l}\text { Cross-sectional } \\
\text { study }\end{array}$ & NA & NA & $\begin{array}{l}\text { Obese female patients with a BMI value } \\
\text { above } 35 \mathrm{~kg} / \mathrm{m}^{2} \text { who underwent bariatric } \\
\text { surgery. BMI, AST, ALT, GGT higher in } \\
\text { case than control; FBG, total-c, AP, HDL-c, } \\
\text { TG, albumin, bilirubin similar in case and } \\
\text { control. }\end{array}$ & $\begin{array}{l}22 \\
(Q, S)\end{array}$ \\
\hline Kalhan SC, $2011^{(26)}$ & USA & $\begin{array}{c}35 \\
(14 / 21,44)\end{array}$ & $\begin{array}{c}25 \\
(7 / 18,43)\end{array}$ & $\begin{array}{l}\text { Cross-sectional } \\
\text { study }\end{array}$ & 11 & 24 & $\begin{array}{l}\text { Non diabetic subjects. BMI, insulin, FBG, } \\
\text { HOMA-IR, ALT, AST, TG, LDL-c higher in } \\
\text { case than control; HDL-C, glutathione } \\
\text { lower in case than control. }\end{array}$ & $\begin{array}{l}23 \\
(P)\end{array}$ \\
\hline Leach NV, 2014(28) & Romania & $\begin{array}{c}50 \\
(35 / 15,46)\end{array}$ & $\begin{array}{c}30 \\
(20 / 10,45)\end{array}$ & $\begin{array}{l}\text { Case-control } \\
\text { study }\end{array}$ & 0 & 50 & $\begin{array}{l}\text { BMI, WC, WHR, presence of MS, SBP, } \\
\text { DBP, insulin, FBG, HOMA-IR, ALT, AST, } \\
\text { TG, total-c, LDL-c higher in case than } \\
\text { control; HDL-c lower in case than control. }\end{array}$ & $\begin{array}{l}23 \\
(J)\end{array}$ \\
\hline Polyzos SA, 2012(27) & Greece & $\begin{array}{c}31 \\
(8 / 23,54)\end{array}$ & $\begin{array}{c}22 \\
(5 / 17,52)\end{array}$ & $\begin{array}{l}\text { Cross-sectional } \\
\text { study }\end{array}$ & 15 & 16 & $\begin{array}{l}\text { BMI, SBP, DBP, AST, ALT, AST/ALT ratio, } \\
\text { GGT, insulin, FBG, HOMA-IR, TG, ferritin } \\
\text { higher in case than control; HDL-c lower } \\
\text { in case than control; LDL-C, total-c similar } \\
\text { in case and control. }\end{array}$ & $\begin{array}{l}23 \\
(J)\end{array}$ \\
\hline Sazci A, $2008^{(29)}$ & Turkey & $\begin{array}{c}57 \\
(31 / 26,44)\end{array}$ & $\begin{array}{c}245 \\
(106 / 139,46)\end{array}$ & $\begin{array}{l}\text { Case-control } \\
\text { study }\end{array}$ & 0 & 57 & $\begin{array}{l}\text { BMI, WC, inssulin, FBG, ALT, AST, bilirubin, } \\
\text { total-c, TG, LDL-C, VLDL-C, ferritin higher } \\
\text { in case than control; HDL-C, LDH, albumin } \\
\text { similar in case and control. }\end{array}$ & $(J, P, V, Y)$ \\
\hline
\end{tabular}

NAFL, non-alcoholic fatty liver; NASH, non-alcoholic steatohepatitis; NA, not available; BMI, body mass index; AST, aspartate aminotransferase; ALT, alanine aminotransferase; GGT, $\gamma$-glutamyltransferase; AP, alkaline phophatase; HOMA-IR, homeostasis model assessment of the insulin resistance index; TG, triglycerides; HDL-C, high-density-lipoprotein cholesterol; total-c, total cholesterol; LDL-C, low-density-lipoprotein cholesterol; FBG, fasting blood glucose; VLDL-C, very low-density-lipoprotein cholesterol; WC, waist circumference; WHR, waist/hip ratio; MS, metabolic syndrome; SBP, systolic blood pressure; DBP, diastolic blood pressure; LDH, lactate dehydrogenase. Quality was assessed by the modified 24-item STROBE score, with the item(s) not satisfied by the study indicated in parentheses: (A) Title and abstract informative and balanced; (B) Background/rationale stated in the introduction; (C) Objective(s) specified in the introduction; (D) Study design correctly and presented early in the paper; (E) Setting, locations, and relevant dates described; (F) Eligibility criteria, methods of selection and follow-up described; (G) Diagnostic criteria, outcomes, exposures, predictors, potential confounders, and effect modifiers for all variables clearly defined; $(\mathrm{H})$ Sources of data and details of methods of measurement given for each variable of interest; (I) Any efforts to address potential sources of bias described; (J) How the study size was arrived at clearly explained; (K) How quantitative variables were handled in the analyses clearly explained; (L) All statistical methods, how missing data and loss to follow-up were addressed, any sensitivity analyses clearly described; (M) Numbers of individuals at each stage of study reported; (N) Characteristics of study participants, number of participants with missing data, average and total follow-up time clearly described; (O) Outcome events or summary measures over time reported; (P) Unadjusted and confounder-adjusted estimates and their precision (e.g., 95\% CI) reported; (Q) Analyses of subgroups and interactions, and sensitivity analyses reported; $(R)$ Key results with reference to study objectives summarized; (S) Limitations of the study discussed; ( $T$ ) Cautious overall interpretation of results given; $(U)$ Generalizability (external validity) of the study results discussed; (V) Source of funding and role of the funders described; Two specifically incorporated items: (Y) Blinding of pathologists reading liver biopsy; (Z) Liver biopsy processed and scored according to standard criteria.

Table 2. Main outcome measures of studies included in the meta-analyses

\begin{tabular}{|c|c|c|c|c|c|c|}
\hline \multirow{2}{*}{ Study, year } & \multicolumn{2}{|c|}{ Homocysteine ( $\mu \mathrm{mol} / \mathrm{L})$ mean (SD) } & \multicolumn{2}{|c|}{ Folate (nmol/L) mean (SD) } & \multicolumn{2}{|c|}{ Vitamin B12 (pmol/L) mean (SD) } \\
\hline & Case & Control & Case & Control & Case & Control \\
\hline de Carvalho SC, $2013^{(22)}$ & $9.69(2.89)$ & $8.49(1.76)$ & $15.25(3.27)$ & $15.12(3.02)$ & $473.11(199.40)$ & $355.02(178.04)$ \\
\hline Franco Brochado MJ, 2013(23) & $\begin{array}{l}11(6) \\
(101)^{*}\end{array}$ & $\begin{array}{l}8(2) \\
(109)^{*}\end{array}$ & $\begin{array}{c}27.24(13.62) \\
(70)^{*}\end{array}$ & $\begin{array}{c}27.24(13.62) \\
(65)^{*}\end{array}$ & $\begin{array}{c}468.53(203.23) \\
(70)^{*}\end{array}$ & $\begin{array}{c}294.12(131.54) \\
(67)^{\star}\end{array}$ \\
\hline Gulsen M, 2005(24) & $13.77(2.65)$ & $10.50(1.53)$ & $9.79(2.58)$ & $13.91(3.31)$ & $277.21(6.54)$ & $345.37(76.56)$ \\
\hline Hirsch S, $2005^{(25)}$ & $9.18(2.40)$ & $9.65(3.91)$ & $21.1(7.9)$ & $27.7(7.04)$ & $300.5(210.7)$ & $286.4(144.1)$ \\
\hline Kalhan SC, 2011(26) & $\begin{array}{c}9.0(2.1) \\
(3)^{\#}\end{array}$ & $\begin{array}{c}7.3(2.0) \\
(0)^{\#}\end{array}$ & NA & NA & NA & NA \\
\hline Leach NV, 2014 & $12.73(3.43)$ & $9.95(2)$ & $211.4(117.4)$ & $215.6(117.4)$ & $223.5(96.0)$ & $171.9(64.3)$ \\
\hline Polyzos SA, 2012(27) & $\begin{array}{c}13.5(2.6) \\
(23)^{\#}\end{array}$ & $\begin{array}{c}12.6(4.6) \\
(8)^{\#}\end{array}$ & $26.33(12.03)$ & $22.47(8.91)$ & $286.73(102.72)$ & $254.22(81.29)$ \\
\hline Sazci A, 2008 $8^{(29)}$ & $13.21(7.42)$ & $10.48(3.06)$ & $8.75(3.13)$ & $8.49(3.85)$ & $346.37(195.10)$ & $246.33(86.89)$ \\
\hline
\end{tabular}

SD, standard deviation; NA, not available. *Values in parentheses represent number of patients. \#Values in parentheses represent number of patients with hyperhomocysteinemia (defined as homocystein $\geq 12.0 \mu \mathrm{mol} / \mathrm{L}$ ). 


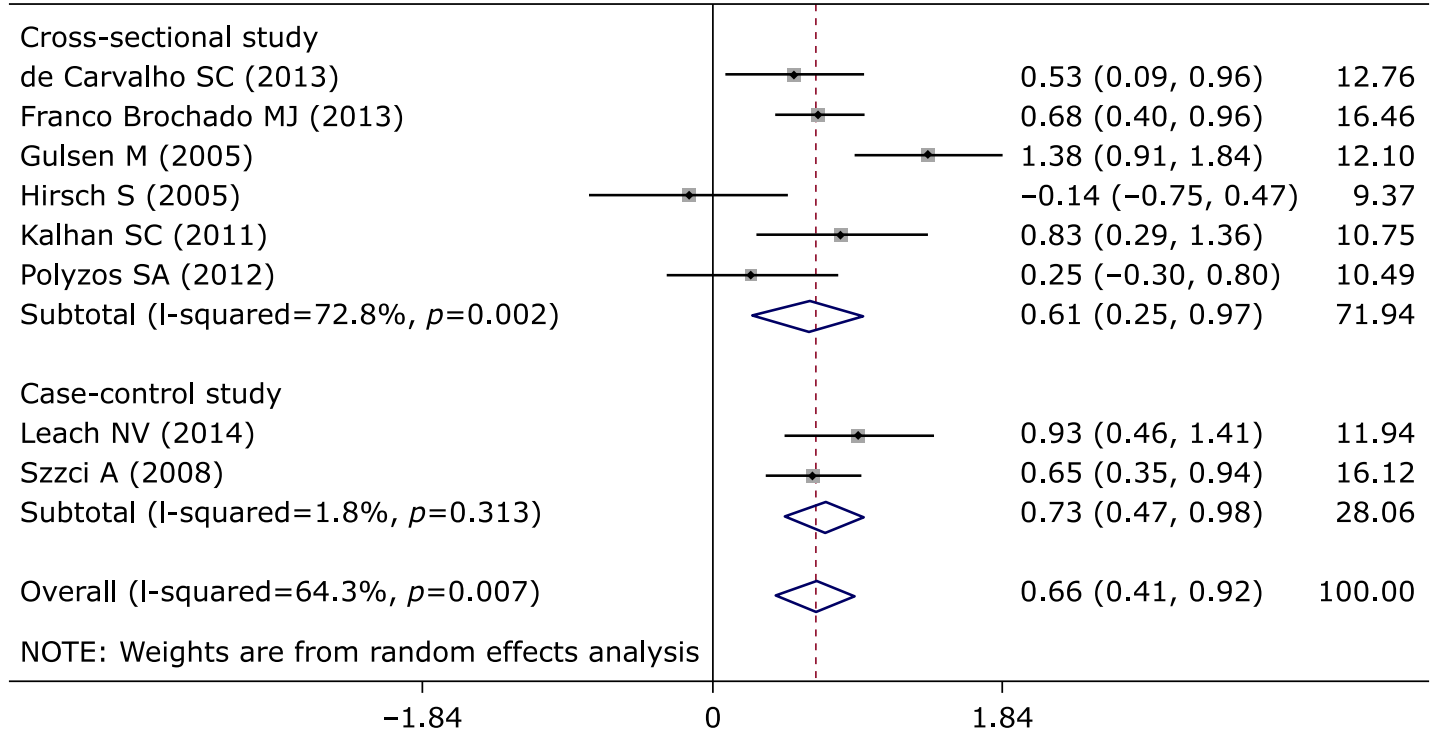

Fig. 2. Meta-analysis of homocysteine levels in NAFLD vs controls. Forest plot demonstrated higher homocysteine level in NAFLD compared to controls. SMD, standard mean difference; Cl, confidence interval; NAFLD, non-alcoholic fatty liver disease.

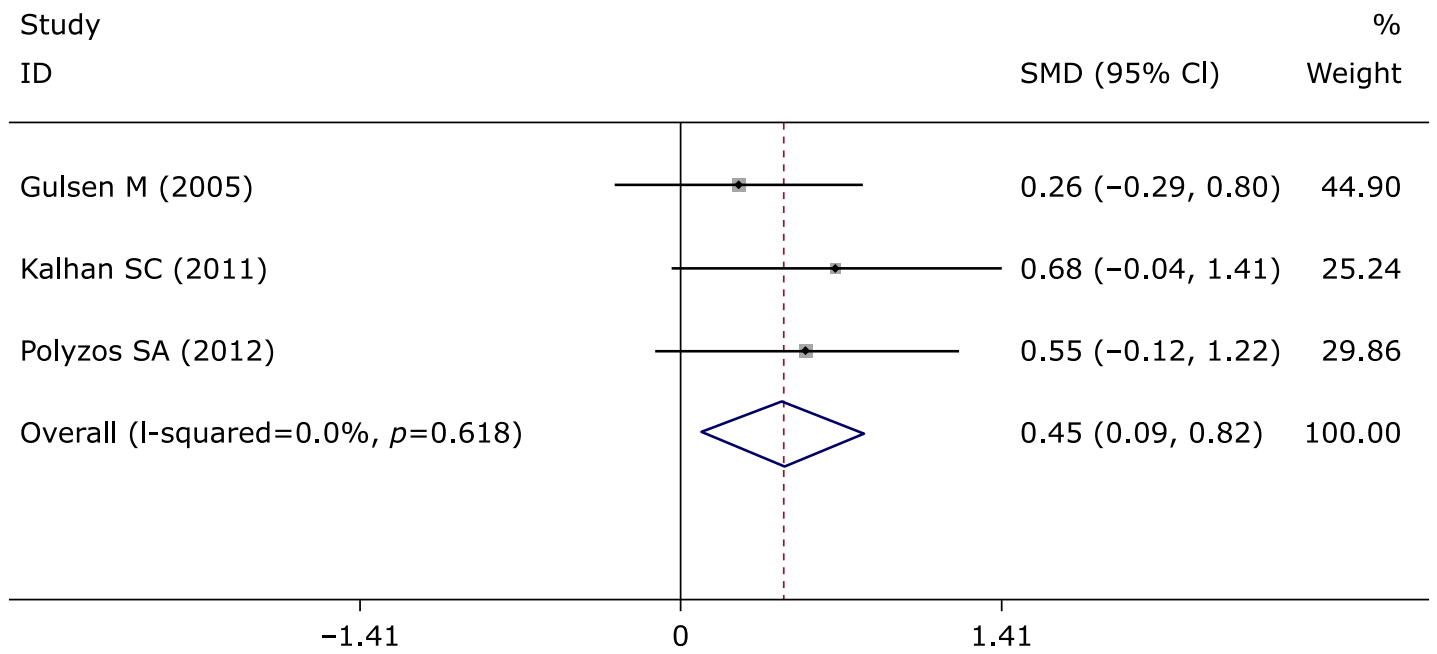

Fig. 3. Meta-analysis of homocysteine levels in NAFL patients vs controls. Forest plot demonstrated higher homocysteine level in NAFL patients than controls. SMD, standard mean difference; $\mathrm{Cl}$, confidence interval.

We considered hyperhomocysteinemia when Hcy level resulted $\geq 12.0 \mu \mathrm{mol} / \mathrm{L}$. Based on the cut-off value, NAFLD participants were associated with a significant increased risk of hyperhomocysteinemia (OR 5.09, 95\% CI: $1.69,15.32)\left(I^{2}=0.0 \%, p=0.958\right)$ compared with the controls (Fig. 5).

Folate level. Seven studies ${ }^{(22-25,27-29)}$ including 800 subjects (331 NAFLD cases and 469 controls) measured plasma folate level in NAFLD subjects and healthy controls. There was neither significant difference of folate level between NAFLD subjects and healthy controls overall (SMD: $-0.26 \mathrm{nmol} / \mathrm{L}, 95 \% \mathrm{CI}:-0.69$, $0.17 \mathrm{nmol} / \mathrm{L})\left(I^{2}=85.7 \%, p<0.01\right)$, nor in subgroup analysis (Fig. 6).

Vitamin B12 level. There were totally 802 participants (331 NAFLD cases and 471 controls) enrolled in seven studies, ${ }^{(22-25,27-}$ ${ }^{29)}$ which provided data regarding plasma vitamin B12 level in
NAFLD cases and controls. Similarly, there was no significant difference of vitamin B12 level between NAFLD subjects and healthy controls (SMD: $0.28 \mathrm{pmol} / \mathrm{L}, 95 \% \mathrm{CI}:-0.35,0.92 \mathrm{pmol} /$ L) $\left(I^{2}=93.4 \%, p<0.01\right)$ (Fig. 7). However, subgroup analysis of case-control studies indicated $0.79 \mathrm{pmol} / \mathrm{L}$ higher levels of vitamin B12 (95\% CI: $0.54,1.04 \mathrm{pmol} / \mathrm{L})\left(I^{2}=0.0 \%, p=0.342\right)$ in NAFLD group than control group.

Sensitivity analyses. We performed sensitivity analyses to investigate the influence of each study on the overall effect. The exclusion of any individual study did not markedly affect the overall measures of the association, except for the exclusion of the study by Gulsen, ${ }^{(24)}$ after which the SMD for vitamin B12 level changed to $0.66 \mathrm{pmol} / \mathrm{L}$ (95\% CI: $0.41,0.91 \mathrm{pmol} / \mathrm{L}$ ).

Publication bias. The shapes of the funnel plots for the studies on Hcy levels (Supplemental Fig. 1*), folate levels (Supplemental 


\begin{tabular}{l|rrr}
\hline Gulsen M (2005) & $2.74(2.11,3.37)$ & 19.23 \\
Kalhan SC (2011) & $0.92(0.33,1.51)$ & 19.57 \\
Leach NV (2014) & $0.93(0.46,1.41)$ & 20.47 \\
Polyzos SA (2012) & $-0.08(-0.72,0.57)$ & 19.12 \\
Sazci A (2008) & $0.65(0.35,0.94)$ & 21.60 \\
Overall (I-squared $=91.0 \%, p=0.000)$ & $1.02(0.28,1.76)$ & 100.00 \\
NOTE: Weights are from random effects analysis & -3.37 & 3.37
\end{tabular}

Fig. 4. Meta-analysis of homocysteine levels in NASH vs controls. Forest plot demonstrated higher homocysteine level in NASH than controls. SMD, standard mean difference; $\mathrm{Cl}$, confidence interval; $\mathrm{NASH}$, non-alcoholic steatohepatitis.

Study

$\%$

ID

OR $(95 \% \mathrm{Cl}) \quad$ Weight

\begin{tabular}{l|lll}
\hline Kalhan SC (2010) & $5.49(0.27,111.23)$ & 13.41 \\
Polyzos SA (2012) & $5.03(1.54,16.44)$ & 86.59 \\
Overall (I-squared $=0.0 \%, p=0.958)$ & $5.09(1.69,15.32)$ & 100.00 \\
\hline .00899 & & & \\
\hline
\end{tabular}

Fig. 5. Meta-analysis between NAFLD and hyperhomocysteinemia. Forest plot demonstrated higher risk of hyperhomocysteinemia in NAFLD than controls. OR, odds ratio; $\mathrm{Cl}$, confidence interval; NAFLD, non-alcoholic fatty liver disease.

Fig. 2*), and vitamin B12 levels (Supplemental Fig. 3*) seemed not symmetrical. However, results of Egger's and Begg's tests gave no evidence of significant bias in the included studies (Hcy levels: Egger's tests, $p=0.698$, Begg's tests, $p=0.266$; folate levels: Egger's tests, $p=0.344$, Begg's tests, $p=0.230$; vitamin B12 levels: Egger's tests, $p=0.215$, Begg's tests, $p=0.035)$.

\section{Discussion}

In this meta-analysis, we found NAFLD participants had $0.66 \mu \mathrm{mol} / \mathrm{L}$ higher levels of Hcy $(95 \% \mathrm{CI}: 0.41,0.92 \mu \mathrm{mol} / \mathrm{L})$, and were 5.09 times more likely to have hyperhomocysteinemia (95\% CI: 1.69, 15.32) compared to their controls. The results suggested that there was an association between plasma Hcy levels and patients with NAFLD.

In the past decades, there were a series of experimental studies that verified the association between NAFLD and hyperhomocysteinemia. Bravo et al. ${ }^{(30)}$ found high-fat-diet-induced NAFLD in rats was associated with hyperhomocysteinemia. The hepatic activities of cystathionine $\beta$-synthase (CBS) and cystathionine $\gamma$ lyase (CGS), the enzymes responsible for the breakdown of homocysteine to cysteine via the transsulphuration pathway in the liver, were reduced in the high fat-fed group.

In terms of the potential mechanisms linking Hcy and NAFLD, there are several hypotheses. IR, a key risk factor in the pathogenesis of NAFLD, is linked to the development of oxidative stress and lipotoxicity. A variety of studies have demonstrated that elevated Hcy is associated with IR. ${ }^{(31,32)}$ Fonseca et al. ${ }^{(33)}$ found plasma Hcy concentrations were regulated by acute hyperinsulinemia in nondiabetic but not T2DM subjects, suggesting that IR may contribute to the development of hyperhomocysteinemia. Furthermore, higher plasma Hcy is responsible for disturbed plasma lipids and lipid accumulation in tissues, resulting in fatty liver. ${ }^{(12,34)}$ In addition, Hcy-induced endoplasmic reticulum stress causes dysregulation of the endogenous sterol response pathway, explaining the development of hepatic steatosis observed in hyperhomocysteinemia. ${ }^{(13,35-37)}$ Moreover, direct toxicity of Hcy to 


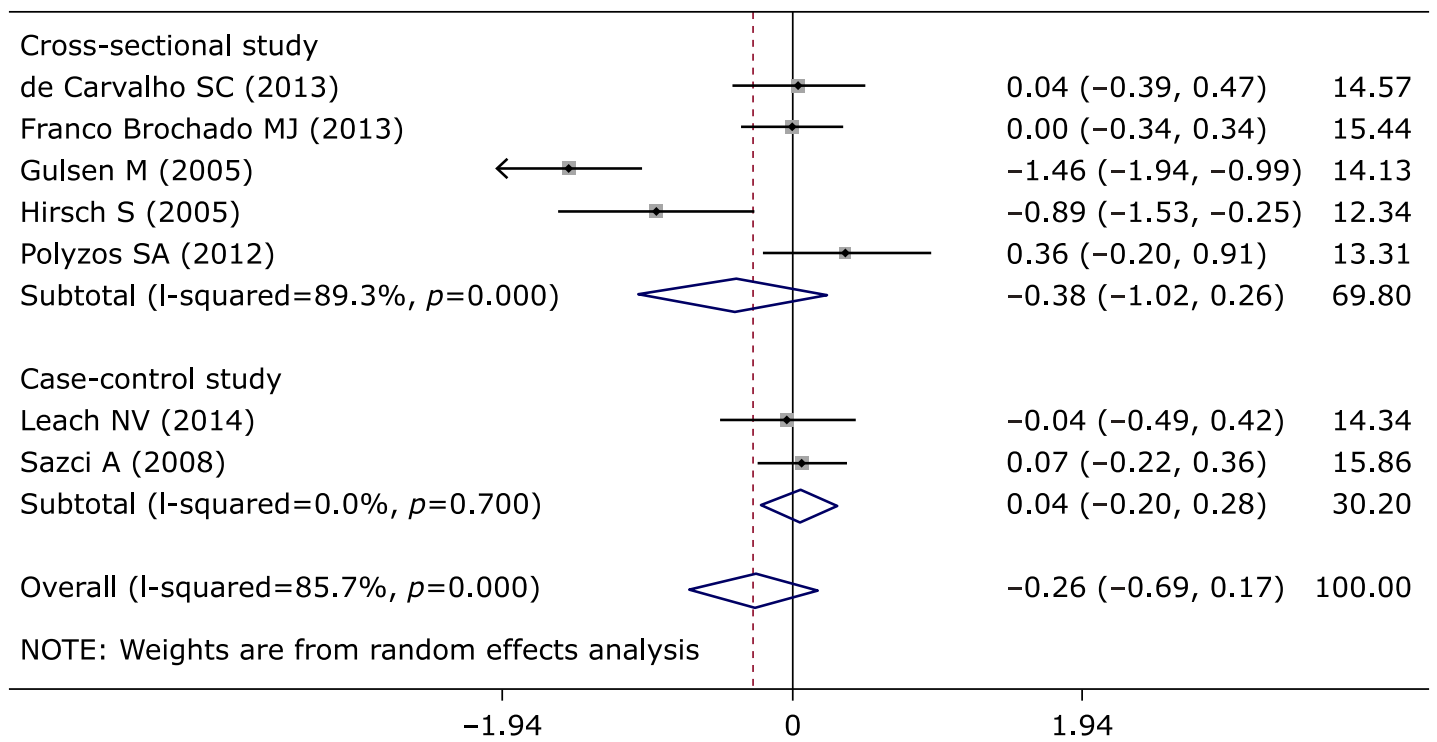

Fig. 6. Meta-analysis of folate levels in NAFLD vs controls. Forest plot demonstrated no significant difference of folate levels in the comparison. $\mathrm{SMD}$, standard mean difference; $\mathrm{Cl}$, confidence interval; NAFLD, non-alcoholic fatty liver disease.

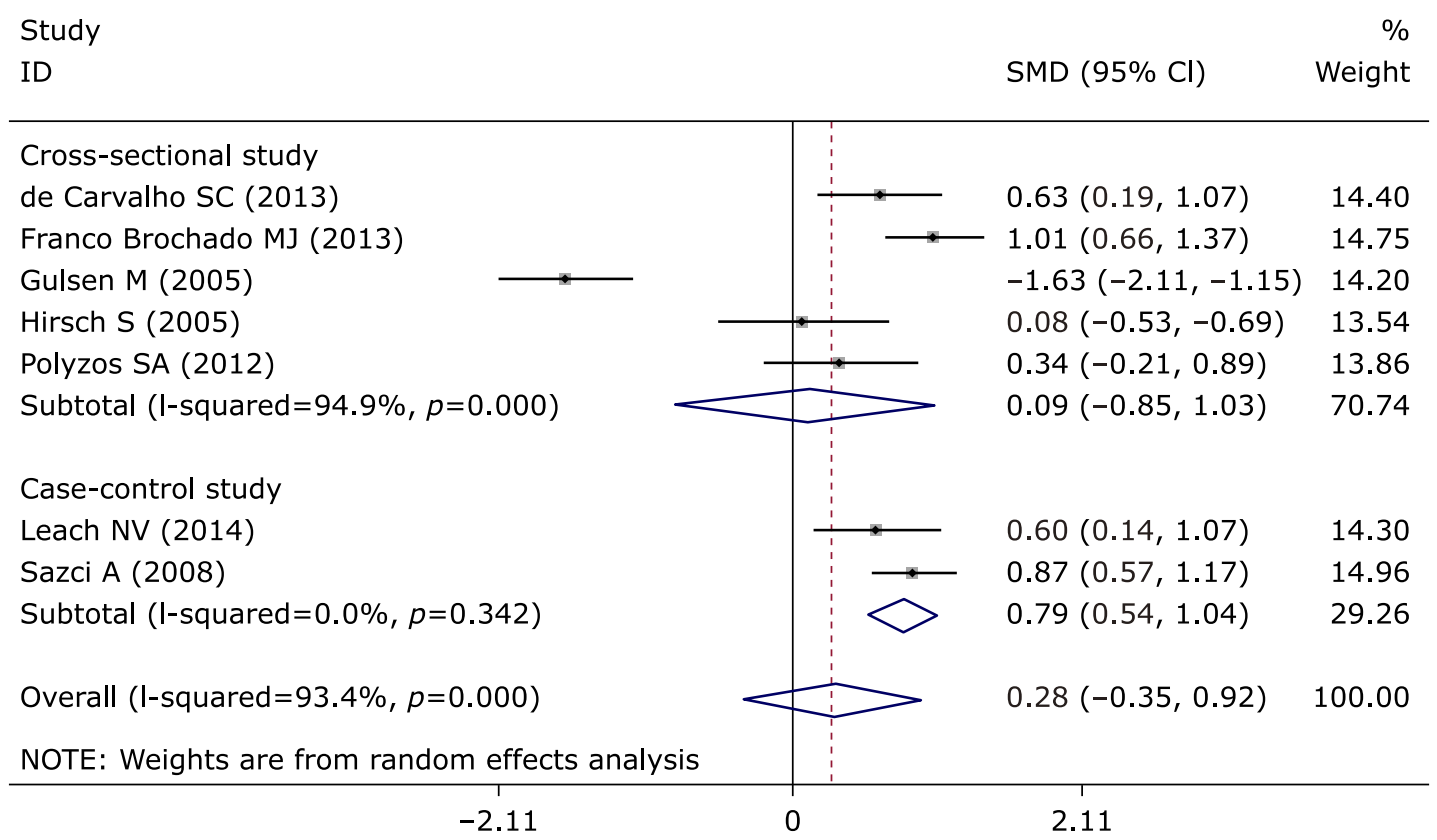

Fig. 7. Meta-analysis of vitamin B12 levels in NAFLD vs controls. Forest plot demonstrated no significant difference of vitamin B12 levels in the comparison. SMD, standard mean difference; Cl, confidence interval; NAFLD, non-alcoholic fatty liver disease.

endothelial injury ${ }^{(38,39)}$ results in microthrombosis in hepatic sinus, which may also explain the pathogenetic role of hyperhomocysteinemia in NAFLD.

Even though this study failed to present statistically significant associations between NAFLD and plasma concentrations of Hcyrelated vitamins, it cannot be denied that folate and vitamin B12 supplementation may have a therapeutic role in patients with hyperhomocysteinemia. Previous trials have revealed that certain doses of vitamins administration may reduce Hcy levels, and thus prevent arteriosclerotic vascular disease. ${ }^{(5,7,40)}$ Recently, in a crosssectional study among 348 Korean adults, ${ }^{(41)}$ the intake of folate had a tendency to decrease NAFLD (diagnosed by ultrasound) risk in male subjects $(\mathrm{OR}=3.37, p=0.077)$. Although it was still unclear, we believe the question whether down-regulation of plasma Hcy levels will lower the risk of NAFLD may be critical in the prevention of NAFLD. In addition, an open-label study in $\mathrm{USA}^{(42)}$ involving ten patients with biopsy-proven NASH showed no significant biochemical improvement after six-month-therapy 
with folate at a dose of $1 \mathrm{mg} /$ day. Obviously, further research is required to examine the efficacy of supplementary Hcy-related vitamins for patients who suffered from NAFLD.

There were many strengths regarding to the present study. To our knowledge, this is the first meta-analysis to evaluate the association of Hcy levels with NAFLD, via a comprehensive search of literature to identify studies published before January 2015. Due to the relatively insensitivity of ultrasound or liver enzyme elevations to detect NAFLD, which may lead to possible misclassification of individuals with NAFLD as healthy controls, we only included NAFLD cases confirmed by liver biopsy. Because NAFLD is a broad spectrum of disease, a subgroup analysis was performed to reveal altered plasma Hcy concentrations in NAFL and NASH, compared to healthy controls. On the other hand, as higher level of Hcy is often established in old age, the mean age of involved subjects was similar in case and control groups. In addition, Hcy and related vitamin levels in NAFLD and healthy controls were estimated separately according to study types. Furthermore, we also examined the risk of hyperhomocysteinemia based on NAFLD status.

However, our study had a few limitations. First of all, most of the original studies did not adjust for the potential confounders, such as body mass index, IR, liver enzymes and hyperlipidemia. We failed to confirm the independence of Hcy as a risk factor for NAFLD. Secondly, the levels of Hcy and related vitamins were measured in confirmed cases of NAFLD, which prevented us

\section{References}

1 Chalasani N, Younossi Z, Lavine JE, et al. The diagnosis and management of non-alcoholic fatty liver disease: practice guideline by the American Gastroenterological Association, American Association for the Study of Liver Diseases, and American College of Gastroenterology. Gastroenterology 2012; 142: 1592-1609.

2 Bugianesi E, Leone N, Vanni E, et al. Expanding the natural history of nonalcoholic steatohepatitis: from cryptogenic cirrhosis to hepatocellular carcinoma. Gastroenterology 2002; 123: 134-140.

3 Méndez-Sánchez N, Arrese M, Zamora-Valdés D, Uribe M. Current concepts in the pathogenesis of nonalcoholic fatty liver disease. Liver Int 2007; 27: 423-433.

4 Selhub J. Homocysteine metabolism. Annu Rev Nutr 1999; 19: 217-246.

5 Boushey CJ, Beresford SA, Omenn GS, Motulsky AG. A quantitative assessment of plasma homocysteine as a risk factor for vascular disease. Probable benefits of increasing folic acid intakes. JAMA 1995; 274: 1049-1057.

6 Homocysteine Studies Collaboration. Homocysteine and risk of ischemic heart disease and stroke: a meta-analysis. JAMA 2002; 288: 2015-2022.

7 Ji Y, Tan S, Xu Y, et al. Vitamin B supplementation, homocysteine levels, and the risk of cerebrovascular disease: a meta-analysis. Neurology 2013; 81: 1298-1307.

8 Yang J, Hu X, Zhang Q, Cao H, Wang J, Liu B. Homocysteine level and risk of fracture: a meta-analysis and systematic review. Bone 2012; 51: 376-382.

9 Huang T, Ren J, Huang J, Liu D. Association of homocysteine with type 2 diabetes: a meta-analysis implementing Mendelian randomization approach. BMC Genomics 2013; 14: 867.

10 Jager A, Kostense PJ, Nijpels G, et al. Serum homocysteine levels are associated with the development of (micro)albuminuria: the Hoorn study. Arterioscler Thromb Vasc Biol 2001; 21: 74-81.

11 Mehedint MG, Zeisel SH. Choline's role in maintaining liver function: new evidence for epigenetic mechanisms. Curr Opin Clin Nutr Metab Care 2013; 16: $339-345$.

12 Obeid R, Herrmann W. Homocysteine and lipids: S-adenosyl methionine as a key intermediate. FEBS Lett 2009; 583: 1215-1225.

13 Werstuck GH, Lentz SR, Dayal S, et al. Homocysteine-induced endoplasmic reticulum stress causes dysregulation of the cholesterol and triglyceride biosynthetic pathways. J Clin Invest 2001; 107: 1263-1273.

14 Liberati A, Altman DG, Tetzlaff J, et al. The PRISMA statement for reporting systematic reviews and meta-analyses of studies that evaluate health care interventions: explanation and elaboration. J Clin Epidemiol 2009; 62: e1-e34. from revealing the causality of hyperhomocysteinemia in NAFLD. A series of questions have been raised. For instance, dose Hcy play a role in the development of NAFLD? Is Hcy qualified for a predictor for the presence or the progression of NAFLD? Further prospective studies are needed to demonstrate a causal role of hyperhomocysteinemia in NAFLD. Thirdly, statistical heterogeneity was observed in some analyses, but the included studies suggested no major clinical heterogeneity and, as a result, we thought appropriate to provide the summarized analyses. Furthermore, the number of studies concerning NAFLD with hyperhomocysteinemia risk was limited. Lastly, we failed to perform subgroup analyses to reveal the effects of other potential factors, e.g., gender and race, on the plasma level of Hcy, owing to insufficient data.

In conclusion, the study demonstrated that NAFLD patients presented increased serum level of Hcy, and were associated with higher risk of hyperhomocysteinemia. Nevertheless, additional studies are needed to determine the causal role of Hcy in the development of NAFLD, and to investigate how Hcy takes part in the pathogenesis of NAFLD. We believe that a better understanding of hyperhomocysteinemia in NAFLD might provide insights into clinical therapy.

\section{Conflict of Interest}

No potential conflicts of interest were disclosed.

15 von Elm E, Altman DG, Egger M, et al. The Strengthening the Reporting of Observational Studies in Epidemiology (STROBE) statement: guidelines for reporting observational studies. Ann Intern Med 2007; 147: 573-577.

16 Kleiner DE, Brunt EM, Van Natta M, et al. Design and validation of a histological scoring system for nonalcoholic fatty liver disease. Hepatology 2005; 41: 1313-1321.

17 Higgins JP, Thompson SG. Quantifying heterogeneity in a meta-analysis. Stat Med 2002; 21: 1539-1558.

18 Higgins JP, Thompson SG, Deeks JJ, Altman DG. Measuring inconsistency in meta-analyses. BMJ 2003; 327: 557-560.

19 Thornton A, Lee P. Publication bias in meta-analysis: its causes and consequences. J Clin Epidemiol 2000; 53: 207-216.

20 Egger M, Davey Smith G, Schneider M, Minder C. Bias in meta-analysis detected by a simple, graphical test. BMJ 1997 ; 315: 629-634.

21 Begg CB, Mazumdar M. Operating characteristics of a rank correlation test for publication bias. Biometrics 1994; 50: 1088-1101.

22 de Carvalho SC, Muniz MT, Siqueira MD, et al. Plasmatic higher levels of homocysteine in non-alcoholic fatty liver disease (NAFLD). Nutr J 2013; 12: 37.

23 Franco Brochado MJ, Domenici FA, Candolo Martinelli Ade L, et al. Methylenetetrahydrofolate reductase gene polymorphism and serum homocysteine levels in nonalcoholic fatty liver disease. Ann Nutr Metab 2013; 63: 193-199.

24 Gulsen M, Yesilova Z, Bagci S, et al. Elevated plasma homocysteine concentrations as a predictor of steatohepatitis in patients with non-alcoholic fatty liver disease. J Gastroenterol Hepatol 2005; 20: 1448-1455.

25 Hirsch S, Poniachick J, Avendaño M, et al. Serum folate and homocysteine levels in obese females with non-alcoholic fatty liver. Nutrition 2005; 21: 137-141.

26 Kalhan SC, Guo L, Edmison J, et al. Plasma metabolomic profile in nonalcoholic fatty liver disease. Metabolism 2011; 60: 404-413.

27 Polyzos SA, Kountouras J, Patsiaoura K, et al. Serum homocysteine levels in patients with nonalcoholic fatty liver disease. Ann Hepatol 2012; 11: 68-76.

28 Leach NV, Dronca E, Vesa SC, et al. Serum homocysteine levels, oxidative stress and cardiovascular risk in non-alcoholic steatohepatitis. Eur J Intern Med 2014; 25: 762-767.

29 Sazci A, Akpinar G, Aygun C, Ergul E, Senturk O, Hulagu S. Association of apolipoprotein E polymorphisms in patients with non-alcoholic steatohepatitis. Dig Dis Sci 2008; 53: 3218-3224.

30 Bravo E, Palleschi S, Aspichueta P, et al. High fat diet-induced non alcoholic 
fatty liver disease in rats is associated with hyperhomocysteinemia caused by down regulation of the transsulphuration pathway. Lipids Health Dis 2011; 10: 60 .

31 Meigs JB, Jacques PF, Selhub J, et al. Fasting plasma homocysteine levels in the insulin resistance syndrome: the Framingham offspring study. Diabetes Care 2001; 24: 1403-1410.

32 Nafiye Y, Sevtap K, Muammer D, Emre O, Senol K, Leyla M. The effect of serum and intrafollicular insulin resistance parameters and homocysteine levels of nonobese, nonhyperandrogenemic polycystic ovary syndrome patients on in vitro fertilization outcome. Fertil Steril 2010; 93: 1864-1869.

33 Fonseca VA, Mudaliar S, Schmidt B, Fink LM, Kern PA, Henry RR. Plasma homocysteine concentrations are regulated by acute hyperinsulinemia in nondiabetic but not type 2 diabetic subjects. Metabolism 1998; 47: 686-689.

34 Noga AA, Stead LM, Zhao Y, Brosnan ME, Brosnan JT, Vance DE. Plasma homocysteine is regulated by phospholipid methylation. J Biol Chem 2003; 278: $5952-5955$.

35 Dara L, Ji C, Kaplowitz N. The contribution of endoplasmic reticulum stress to liver diseases. Hepatology 2011; 53: 1752-1763.

36 Aissa AF, Tryndyak V, de Conti A, et al. Effect of methionine-deficient and methionine-supplemented diets on the hepatic one-carbon and lipid metabolism in mice. Mol Nutr Food Res 2014; 58: 1502-1512.

37 Ji C. Dissection of endoplasmic reticulum stress signaling in alcoholic and non-alcoholic liver injury. J Gastroenterol Hepatol 2008; 23 (Suppl 1): S16S24.

38 Wall RT, Harlan JM, Harker LA, Striker GE. Homocysteine-induced endothelial cell injury in vitro: a model for the study of vascular injury. Thromb Res 1980; 18: 113-121.

39 Blann AD. Endothelial cell damage and homocysteine. Atherosclerosis 1992; 94: 89-91.

40 Yi X, Zhou Y, Jiang D, Li X, Guo Y, Jiang X. Efficacy of folic acid supplementation on endothelial function and plasma homocysteine concentration in coronary artery disease: a meta-analysis of randomized controlled trials. Exp Ther Med 2014; 7: 1100-1110.

41 Han JM, Jo AN, Lee SM, et al. Associations between intakes of individual nutrients or whole food groups and non-alcoholic fatty liver disease among Korean adults. J Gastroenterol Hepatol 2014; 29: 1265-1272.

42 Charatcharoenwitthaya P, Levy C, Angulo P, Keach J, Jorgensen R, Lindor KD. Open-label pilot study of folic acid in patients with nonalcoholic steatohepatitis. Liver Int 2007; 27: 220-226. 